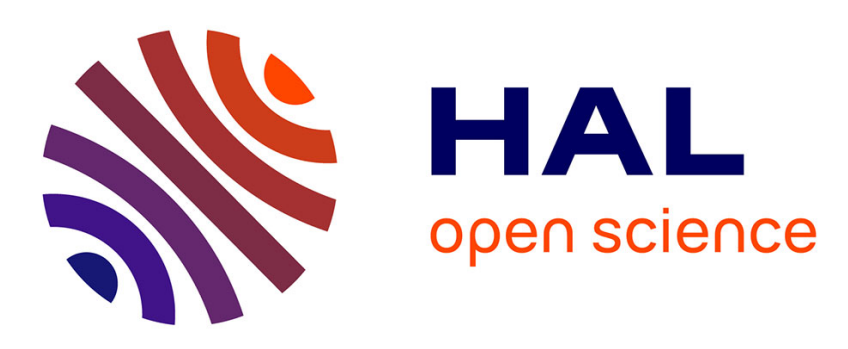

\title{
Life prediction for carbon fibre filament wound composite structures
}

Anthony R. Bunsell, Alain Thionnet

\section{To cite this version:}

Anthony R. Bunsell, Alain Thionnet. Life prediction for carbon fibre filament wound composite structures. Philosophical Magazine, 2010, 90, pp.4129-4146. 10.1080/14786430903575140 . hal00596598

\section{HAL Id: hal-00596598 \\ https://hal.science/hal-00596598}

Submitted on 28 May 2011

HAL is a multi-disciplinary open access archive for the deposit and dissemination of scientific research documents, whether they are published or not. The documents may come from teaching and research institutions in France or abroad, or from public or private research centers.
L'archive ouverte pluridisciplinaire HAL, est destinée au dépôt et à la diffusion de documents scientifiques de niveau recherche, publiés ou non, émanant des établissements d'enseignement et de recherche français ou étrangers, des laboratoires publics ou privés. 


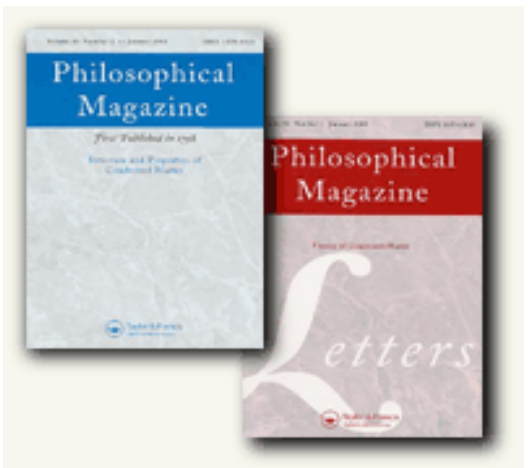

\section{Life prediction for carbon fibre filament wound composite structures}

\begin{tabular}{|c|c|}
\hline Journal: & Philosophical Magazine \& Philosophical Magazine Letters \\
\hline Manuscript ID: & TPHM-09-Jul-0311.R3 \\
\hline Journal Selection: & Philosophical Magazine \\
\hline $\begin{array}{r}\text { Date Submitted by the } \\
\text { Author: }\end{array}$ & 17-Nov-2009 \\
\hline Complete List of Authors: & $\begin{array}{l}\text { Bunsell, anthony; MinesParistech, Materials } \\
\text { Thionnet, Alain; Université de Bourgogne, Mirande-Mechanics }\end{array}$ \\
\hline Keywords: & $\begin{array}{l}\text { carbon fibres, composite materials, computer simulation, } \\
\text { experimental, finite-element modelling, hydrogen, Monte-Carlo, } \\
\text { multiscale modelling }\end{array}$ \\
\hline Keywords (user supplied): & pressure vessels, failure, lifetime prediction \\
\hline
\end{tabular}

\section{今scholarONE" \\ Manuscript Central}


The processes governing the failure of filament wound composite structures have been examined. It is shown that the fibres controlling the failure of such a structure, when it is internally pressurised, can be considered to be subjected only to tensile loads. A multi-scale model has been developed which considers the effects on the scale of the elastic fibres and includes the effects of the viscoelastic matrix as well as debonding around fibre failures. The intact fibres neighbouring fibre breaks are subjected to an increase in stress and a higher probability of failure than elsewhere in the composite. During a monotonic failure test the initially random fibre failures are seen to begin to coalesce in a way governed by the stochastic nature of the fibre breaks and this eventually leads to failure. Under prolonged loading the relaxation of the matrix around fibre breaks causes the overloads in the neighbouring fibres to evolve and to induce delayed fibre breaks which can eventually lead to instability in the structure. The model allows these processes to be taken into account while calculating the behaviour of the whole composite structure.

Key words: Life prediction; composite filament wound structures; fibre failures; viscoelasticity; debonding; pressure vessels.

\section{Introduction}

High performance carbon fibres were first produced commercially in the second half of the 1960s in the UK (1). This was the result of work at the Royal Aircraft Establishment, Farnborough. The carbon fibres were made from polyacrylonitrile precursors and over a period of forty years their composites have become established as one of the most important materials used in advanced aerospace structures. The presently emerging generation of civil aircraft is being made with half their weight made up of carbon fibre reinforced epoxy resin. Today, however, the largest markets for these materials are no longer confined to the aerospace industry but wider industrial sectors are finding uses for these remarkable materials. This success has to no small degree been aided by researchers, such as Anthony Kelly and his colleagues, who have allowed an in-depth understanding of the physical mechanisms governing the behaviour of composites (2).

One of the major challenges, however, is in the prediction of long-term behaviour and reliability of composite structures. This is particularly important as advanced composites are often used in structures the failure of which would be catastrophic. It is only through extending the legacy that an earlier generation of researchers has left to us that techniques based on a true understanding of damage processes in advanced composites can be developed and reliability in assessing composite structures as well as optimising designs can be achieved. One of the most challenging applications is in pressure vessels for the storage of gas at high pressures. 


\section{Figure 1: Natural gas cylinders made from carbon fibre reinforced epoxy resin on the roof of a bus.}

Composites are ideally suited for this application. This is due to their light weight, high strength and rigidity. They are presently used for storing natural gas at pressures usually up to $20 \mathrm{MPa}$ and are actively being investigated for the storage of hydrogen at pressures of 70MPa. However, legislation and common sense require that pressure vessels be tested periodically to ensure their safe continuing use in service. At present, there are no proof testing techniques or in-service reliability assessments techniques which are mentioned in standards that are suitable or based on the failure processes known to control lifetimes of composite structures. The standards which are described are based on the behaviour of metals which fail by processes which do not occur in composites. Composite materials do not fail by simple crack propagation and in these materials, plastic deformation is not a dominant mechanism determining fracture toughness. A hydraulic test on a composite pressure vessel has only one clear outcome, which is that fibres, which ordinarily would not have broken, fail during the test and the vessel is closer to failure than it was before the test, as is illustrated in Figure 2. Visual inspection is not an alternative technique as damage, due to, say an impact, produces delamination on the reverse side of the composite and this is not available for inspection. 


\section{Figure 2: A hydraulic test would greatly increase the number of broken fibres in the pressure vessel.}

There are techniques, such as ultrasonic inspection and others, that permit localised damage due to impact to be identified, however, the pressure vessels will be used for decades, possibly twenty or thirty years and the general assumption that carbon fibre reinforced epoxy resin composites do not age, due to the combined effects of pressure and time is easily shown to be a fallacy. This paper will demonstrate how such composites evolve with pressure and time and propose means to determine residual lifetimes.

Filament wound composite pressure vessels, of the type considered in this study, are made of fine carbon fibres, typically T $\overline{7} \overline{0}$ fibres or equivalent, made from polyacrylonitrile precursors. The fibres have diameters of $7 \mu \mathrm{m}$, they are perfectly elastic with a Young's modulus of 235 GPa and in common with all populations of fibres, show considerable scatter in their strengths, giving a Weibull modulus of typically 6 . The fibres are placed on geodesic paths around a mandrel, which later serves as a liner to ensure gas tightness. This means that when the vessel is pressurised the fibres are subjected only to tensile forces and at the level of the fibres an analogy can be made with the behaviour of a unidirectional composite loaded in the fibre direction. As with a unidirectional composite, failure of the material means that the fibres have to break. In addition the burst strengths of the vessels are controlled by the winding of a fretting layer of fibres over the cylindrical part of the pressure vessel, at $90^{\circ}$ to its major axis. In the case of a carbon fibre composite, the fibres support around $99 \%$ of the load. The failure of carbon fibres is therefore clearly the mechanism which determines the failure of the composite pressure vessel. It is not crack propagation, as in a metal pressure vessel, as is assumed in the standards.

The consequences of failure of fibres in a composite material have been examined in a number of studies, beginning with Cox who developed a 2-D analytical analysis of load sharing between elastic fibres embedded in an elastic matrix (4). This study revealed the fundamental mechanism controlling composite behaviour to be load transfer from the matrix, which undergoes shear near the fibre, to which it is well bonded, to the fibre which experiences tensile force loads which increase from its end. By writing equations of equilibrium between the shear forces in the matrix and the tensile forces in the fibre, Cox was able to provide analytical solutions for the stress states in and around short or discontinuous fibres in a composite. Subsequent studies considered the effect of perfectly plastic matrices and were shown to simplify the analysis (2). Other studies considered the effects of fibre failure on the other unbroken fibres in a composite $(5,6)$. It was shown that the effect of a fibre break in a composite was confined to a small volume of material around the break and that only the fibres immediately neighbouring the fibre break experienced any change of stress. With the availability of computers and increasing computational capacity this situation has been the subject of many subsequent two and three dimensional numerical studies,

| including a few which consider the effects of a viscoelastic matrix (7-9).

Studies in our group have examined this problem with the aim of improving the models and applying the results to various filament wound structures, including pressure vessels. In order to tackle this problem an immediate practical problem has to be considered. In a unidirectional cfrp specimen with a cross section of $20 \mathrm{~mm} \times 1 \mathrm{~mm}$ there are about 136,000 
fibres. In any cross section of a CNG tank there are around 1.25 million fibres. It is clear that any attempt to model the behaviour of a complete structure with a discretisation at the level of the individual fibres would encounter unacceptable computational times. Nevertheless the mechanisms which govern failure occur at the scale of the individual fibres. The $\mathrm{FE}^{2}$ multiscale approach which has been adopted can be seen to share points in common described in references (10 and 11).

\section{Failure process on the microscopic scale}

Studies of the effect of the stochastic nature of fibre failures in composites have underlined the controlling nature of this type of damage (12-14).

The micromechanics study considers a three-dimensional analysis of a representative volume element (RVE) comprising all the processes to be considered. These are fibre strength and its stochastic nature, fibre tensile elastic modulus and the properties of the matrix. Several levels of refinement are considered so that at the simplest level both fibres and matrix are considered to be elastic. Interfacial debonding between the broken fibre and the matrix is considered at a higher level of refinement. Finally, at the highest level of sophistication, the matrix is considered to be viscoelastic, so that its properties are time dependent, as are the effects of load transfer between neighbouring fibres, which are due to shear of the matrix.

In this study both the fibres and the matrix are considered to be homogeneous continua. The orthorhombic spatial frame used at the microscopic level is $\mathrm{R}_{l o c}=(O, \overline{\boldsymbol{x}}, \overline{\mathbf{y}}, \overline{\mathbf{z}})$ for which $(x, y$, $z$ ) describe the coordinates of a point $M$. The vector $\overline{\mathbf{z}}$ is aligned parallel to the fibres. Earlier studies have allowed the Representative Volume Element (RVE) to be defined for the undamaged composite (15-18). The original 2D model showed that a constant average unidirectional composite failure stress could be calculated using a RVE consisting of six parallel fibres of $8 \mathrm{~mm}$ length (18). This model has been extended to the 3D case by first considering RVEs consisting of six by six square arrays of fibres with lengths of $8 \mathrm{~mm}$. This has been refined for geometrical considerations to arrays consisting of 32 fibres. The degrees of damage were modelled at six levels of failure in the RVE, written as E-N, in which $\mathrm{N}$ represents the state of damage in the group of thirty two fibres. The values of $\mathrm{N}$ represent the fraction of single broken fibres to unbroken fibres and were $\mathrm{N}=32,16,8,4$ and 2, so that, for example, when $\mathrm{N}$ equals 8 , one in eight fibres is considered broken. The associated periodic cell was given the abbreviation $\mathrm{C}-\mathrm{N}$. The failed state E-0 is represented by $\mathrm{C}-0$. The state which represents isolated fibre breaks, so that no interaction occurs between breaks, is described by the damage state E-32 and gives unvarying failure stress for the composite. This is the justification for choosing the RVE consisting of 32 unbroken fibres as representing three-dimensional state of the undamaged composite structure.

It follows that the composite is considered to be periodic with a hexagonal fibre arrangement in the $(\bar{x}, \bar{y})$ plane, the periodic cell, which is representative of the RVE and called CS32, consisting of cells of 32 fibres. Its geometry is considered to be a parallelepiped with the $\overline{\mathbf{z}}$ axis parallel to the fibres and the section is a square with sides of length c (Fig.3). From reference 15 , the length in the $\overline{\mathbf{z}}$ direction was taken to be between the planes $\mathrm{z}=0$ and $\mathrm{z}=\mathrm{L}$ $=8 \mathrm{~mm}$. The reference origin $O$ is the geometrical centre of the section in the plane $z=0$. At this scale, at point $M$, the stress tensor is described as $\sigma$ and the strain as $\varepsilon$. 
Figure 3: Model of the failure process on the scale of the carbon fibres.

The fibre is always considered to be linearly elastic, homogeneous and isotropic. In contrast, two types of behaviour have been considered for the matrix. The most reductive behaviour considered the matrix to be linearly elastic, homogeneous and isotropic. A more realistic model considers the matrix to be linearly viscoelastic with behaviour described by the following equation: $\dot{\boldsymbol{\sigma}}+\boldsymbol{\gamma} \boldsymbol{\sigma}=\mathbf{C}_{0} \dot{\boldsymbol{\varepsilon}}+\boldsymbol{\gamma} \mathbf{C}_{\infty} \boldsymbol{\varepsilon}$ in which $\gamma$ is the fourth order relaxation tensor; $\mathrm{C}_{0} \underline{\text { is }}$ the initial rigidity tensor and $\bar{C}_{\infty}$ the final rigidity tensor after an infinite time. As the matrix is istoropic, each of the tensors is isotropic. In this way the notion of time can be included in the model of the behaviour of the composite and permits delayed composite failure to be studied.

Earlier studies have shown that this approach accurately describes what happens in the vicinity of a fibre break (15-17) and can account for:

- $\quad$ the random nature of fibre breaks and the points of failure along the fibre length;

- the number of breaks in a RVE at five levels of damage going from the undamaged to the failed state. These states are : C32, C16, C8, C4, C2 (Fig. 2) and contain respectively $1,2,4,8,16$ broken fibres amongst the 32 present in the CS32 cell;

- the load transfer to and along the intact fibres due to the fibre failures.

Debonding is modelled by the undoing of the finite element boundary nodes of the matrix and the broken fibre. Outside this zone the fibre-matrix bond is considered to be perfect. The debonded length is considered to be twice the distance from the broken end of the fibre to the point where the fibre and matrix are again adhered. In this way the model accounts for:

- the effects of interfacial debonding around the fibre break on load transfer to intact fibres. Ten debonding lengths have been considered from 3.5 to $35 \mu \mathrm{m}$ in steps of $3.5 \mu \mathrm{m} ;$

From the above considerations the model considers

- the effects of the viscoelastic nature of the matrix, which is considered to be linear, on load transfer to neighbouring intact fibres.

In addition the present study also takes into account the fibre volume fraction.

The goal of these calculations is to simulate the failure of fibres and to observe the evolution of the axial stress in neighbouring intact fibres as a function of the damage state, time, distance from the plane in which the break lies and as a function of any debonding of the fibre-matrix interface. In order to do this we define a coefficient of load transfer, $\mathrm{k}_{\mathrm{l}}$. This is obtained as the mean of two axial stresses, in the fibre considered, calculated between two consecutive normal sections of the fibre in the FE mesh compared to the same quantity in the undamaged state.

The coefficient of load transfer along the lengths of the fibres is given by:

$$
\boldsymbol{k}_{r}\left(C, d, t, V_{f}, Z\right)=\frac{\int_{\mathbf{Z}_{\mathrm{i}}}^{Z_{\mathrm{i}+1}} \int_{\mathbf{S}_{\mathrm{F}}} \sigma_{z z}\left(\mathbf{C}, d, t, \mathbf{V}_{\mathrm{f}}, x, y, z\right) d x d y d z}{\int_{\mathbf{Z}_{\mathrm{i}}}^{Z_{\mathrm{i}+1}} \int_{\mathbf{S}_{\mathrm{F}}} \sigma_{z z}\left(C S 32, d=0, t=O, V_{f}, x, y, z\right) d x d y d z}
$$

In which

$\mathrm{C}$ is the cell representing the state of damage considered $d$ is the debonded length
Formatted: Lowered by $5 \mathrm{pt}$

Formatted: Subscript

Formatted: Subscript 
It has been verified that the discretisation employed is that which results in convergence. The mesh is so fine that the stresses are nearly constant in each element.

Whether or not there is debonding at the fibre/matrix interface or if the matrix is viscoelastic, or not, the definition for $k_{r}$ remains valid. However, in order to separate the different effects we can write $\mathbf{k}_{\mathbf{r}}=\mathbf{K}_{\mathbf{r}+} \mathbf{K}_{\mathbf{r}}^{\mathbf{d}}+\mathbf{K}_{\mathbf{r}}^{\mathrm{v}}$ where $\mathbf{K}_{\mathbf{r}}$ is the load transfer exclusively due to a fibre break; $\mathbf{K}_{\mathbf{r}}^{\mathbf{d}}$ is the additional part of the load transfer exclusively due to the debonding, with $\mathbf{K}_{\mathbf{r}}^{\mathbf{d}}=0$ if the effect is neglected; and $\mathbf{K}_{\mathbf{r}}^{\mathbf{v}}$ is exclusively due to the viscoelastic properties of the matrix, with $\mathbf{K}_{\mathbf{r}}^{\mathrm{v}}=\mathbf{O}$ if this effect is neglected.

\subsection{A data base at the microscopic scale}

Comparisons have been made with the self consistent model in which one broken fibre is considered embedded in a homogeneous medium having the macroscopic properties of the intact composite (7). The results obtained by the present model, for the case in which interfacial bonding is maintained after failure, are in good agreement with such self consistent models. However, such models inevitably underestimate the stresses on intact fibres near the plane of failure as the homogeneous equivalent material is more rigid than the matrix material (19). This leads to difficulties in understanding the subsequent behaviour of these fibres in a model that takes the effects of time into account. These difficulties are removed in the present analysis which, in the case of a viscoelastic matrix and with data on the stochastic nature of fibre strength, allows the effects of matrix relaxation around fibre breaks to be evaluated. The result is seen as an increase in time, over increasing lengths, of the stresses on intact fibres neighbouring a fibre break. This is the cause of delayed fibre breaks and the evolving accumulation of damage in the composite.

If only one cell is considered and a total debonded length of $70 \mu \mathrm{m}$ taken, as the effect of the fibre break become insignificant beyond this length: the calculation time to obtain geometrical convergence for a periodic homogenisation requires approximately a discretisation for 250,000 nodes and two hours of calculation time for an all elastic model. If this approach is applied to a macroscopic structure, for which the number of Gauss points is in the thousands, the calculation time would be unacceptably long. A less time consuming approach is therefore necessary.

The load transfer function has been smoothed and only the load transfer in the fibre nearest to the fibre break calculated. In order to separate the effects of interfacial debonding and those due to the viscoelastic shear of the matrix, we put $\mathbf{k}_{\mathbf{r}}=\mathbf{K}_{\mathbf{r}}+\mathbf{K}_{\mathbf{r}}^{\mathbf{d}}+\mathbf{K}_{\mathbf{r}}^{\mathrm{v}}$ and $\mathbf{K}_{\mathbf{r}_{2}} \mathbf{K}_{\mathbf{r}}^{\mathbf{d}}$ and $\mathbf{K}_{\mathbf{r}_{\mathrm{v}}}^{\mathrm{v}}$ used as a data base describing the microscopic scale which needs to be taken into account in

\begin{tabular}{l} 
Formatted: Lowered by $5 \mathrm{pt}$ \\
Formatted: Lowered by $5 \mathrm{pt}$ \\
Formatted: Lowered by $5 \mathrm{pt}$ \\
Formatted: Lowered by $5 \mathrm{pt}$ \\
Formatted: Lowered by $5 \mathrm{pt}$ \\
Dermatted: Lowered by $5 \mathrm{pt}$ \\
Deleted: $\mathbb{\mathrm { I }}$ \\
\hline
\end{tabular}

\begin{tabular}{|c|}
\hline Deleted: O \\
\hline Deleted: in which \\
\hline $\begin{array}{l}\text { Deleted: II } \\
\mathbb{I}\end{array}$ \\
\hline $\begin{array}{l}\text { Deleted: is the part of the load transfer } \\
\text { exclusively due to the failure of the fibrefI }\end{array}$ \\
\hline Deleted: If \\
\hline $\begin{array}{l}\text { Deleted: is due to the debond with } \\
\mathbf{K}_{\mathbf{r}}^{\mathbf{d}}=0 \text {, if this is not consideredII } \\
\text { II }\end{array}$ \\
\hline $\begin{array}{l}\text { Deleted: is due exclusively to the } \\
\text { viscoelastic behaviour of the matrix with } \\
\mathbf{K} \underset{\mathbf{r}}{\mathbf{v}}=0 \text { if viscoelasticity is not } \\
\text { considered. II } \\
\text { II } \\
\text { These functions are }\end{array}$ \\
\hline
\end{tabular}


the multiscale $\mathrm{FE}^{2}$ model. Using the overall stress applied on the macroscopic scale, the duration of the load and the state of damage, this data base allows, by linearity, to know the stress state in the components at the microscopic scale. The tensile stress in the fibres can therefore be computed, without resolving the problem at the microscopic scale by the finite element method and the evolution of the damage, consisting of increasing numbers of fibre breaks in the RVE can be evaluated. It is assumed that the failure of a fibre induces a debond length of $35 \mu \mathrm{m}$ which has been shown to result in the greatest stress concentration in neighbouring intact fibres.

In order to compare the relative influences of different physical phenomena, for different fibre volume fractions, six levels of refinement in the model must be considered : the first taking into account only fibre breaks; the second adds the effect of load transfer, through the matrix on intact fibres neighbouring the fibre break; the third includes the effects of fibre/matrix debonding from the point of failure and then the previous three degrees of sophistication are again considered but the viscoelastic nature of the matrix is included.

\section{The use of parallel computation to describe the macroscopic scale}

The carbon fibres used in the specimens and in the pressure vessels had radii of $3.5 \mu \mathrm{m}$ so that the dimensions of the CS32 cell, representing a volume fraction $64 \%$, were $0.044 \mathrm{~mm} \mathrm{x}$ $0.044 \mathrm{~mm} \times 8 \mathrm{~mm}$. The volume of the CS32 cell was $\mathrm{V}_{64 \%}=0.0155 \mathrm{~mm}^{3}$.

Calculations carried out for a laboratory plate specimen have shown that the mesh necessary for convergence can be carried out with finite elements (of the c $3 \mathrm{~d} 8$ type) containing 4 RVEs.

It can be seen that if the cylindrical part of the pressure vessel is $500 \mathrm{~mm}$ in length, has an internal radius of $100 \mathrm{~mm}$ and a composite and wall thickness of $10 \mathrm{~mm}$, assuming a fibre volume fraction of $64 \%$, the finite element mesh would consist approximately of 70 million elements each consisting of four RVEs and as many nodes. This would result in approximately 500 million Gauss points and 200 million of degrees of freedom. A pure FE ${ }^{2}$ calculation would involve 500 million $\times 2=600$ million hours of calculation time. The use of parallel computing can be seen to be indispensible.

So as to reduce the calculation time at the macroscopic scale certain assumptions have been made so as to further simplify the problem when considering the long-term macroscopic behaviour of a unidirectional composite. These hypotheses are based on experimental results and do not affect the quality of the final result.

At the macroscopic scale, the composite can be seen as an equivalent homogeneous material, which can be described within orthorhombic coordinates, b b $=\left(\overrightarrow{\boldsymbol{x}}_{1}, \overrightarrow{\boldsymbol{x}}_{2}, \overrightarrow{\boldsymbol{x}}_{3}\right)$. The $\overrightarrow{\boldsymbol{x}}_{1}$ vector is aligned parallel to the fibre direction. At this scale at the point $M$, the stress tensor is and $\Sigma$ and the strain tensor is E.

The first hypothesis is that the fibre failures are the only mechanism determining the failure of the structure. That is to say that the principal mechanism controlling the failure of a unidirectional composite is fibre failures and that non linear behaviour, seen when such composites are loaded off axis, such as intralaminar cracking and viscoelastic behaviour, are not considered. Secondly it is assumed that, unlike the microscopic behaviour, the viscoelastic effects of the matrix, on the macroscopic scale, are negligible, as is seen experimentally. The 
non linear behaviour of the homogenised material is considered only to be due to fibre failure and reflects the density of broken fibres.

The variation of the elastic modulus of the equivalent homogeneous material as a function of the density of fibre breaks could be solved as a problem of periodic homogenisation. However, an approximation, based on the law of mixtures, which gives an upper bound, gives an acceptable result. Finally at the macroscopic scale and in the $b_{\text {loc }}$ base the behaviour of the unidirectional composite the behaviour of the unidirectional composites is given by $\sum(\mathbf{M})=\mathbf{a}(\mathbf{M}): \mathbf{E}(\mathbf{M})$ with $\boldsymbol{a}(\boldsymbol{M})=\boldsymbol{a}^{0}(\boldsymbol{M})$, where $\boldsymbol{a}^{0}(\boldsymbol{M})$ describes the rigidity tensor of the undamaged material at the point $M$, except $\mathbf{a}_{1111}(\mathbf{M})=\mathbf{a}_{1111}^{0}\left\{1-\frac{\mathbf{N}_{\mathbf{R}}(\mathbf{M})}{\mathbf{N}_{\mathbf{T}}}\right\}$ in which $N_{R}, N_{T}(32$ in our case) are respectively the number of broken fibres and the total number of fibres in the RVE.

As a result of the second assumption it can be seen that it is only the increase in load in the intact fibres neighbouring a fibre break at the microscopic level which is important in determining behaviour.

Also the second hypothesis justifies only the increase in loading parallel to the fibre axis being considered and not in other directions.

\section{Multiscale calculation}

The input data for this calculation are:

at the macroscopic scale: the characteristics of the undamaged composite and the law describing the fall in longitudinal rigidity of the equivalent homogonous material;

at the microscopic scale, for each Gauss point: the random choice of 5 failure stresses according to the Weibull statistics, shown experimentally to describe the stochastic failure strengths of the fibres; the data base allowing the axial stresses supported by the fibres in the RVE to be known and the evolution of the damage from the undamaged state in CS32 to complete failure represented in C2.

The multiscale simplified $\mathrm{FE}^{2}$ calculation is iterative and calculates the state of damage (number of fibres broken), at iterative step $n$, having determined the state of damage at step $n$ $l$ in the following manner:

- a time increment is considered together with or without a change to the macroscopic stress depending on whether an increase in load is made or whether the test is at constant load;

- the finite element calculation at the macroscopic scale determines the macroscopic stress on the composite in the direction of the fibres;

at each Gauss point for the microscopic scale:

- a localisation step - Knowing the macroscopic stress and the damage state of the material, the data base for load transfer is applied so as to calculate the axial stress in the fibres contained in the RVEs. This stress allows the number of broken fibres in the 
Figure 4: Simulated fibre failures in unidirectional composites loaded to $80 \%$ of failure load showing scatter due to the stochastic nature of fibre strengths and also variations in Weibull moduli between the A and B curves.

When these results are compared to experimentally obtained curves, as shown in Figure 5 there is very good agreement. Although, at the microscopic scale and near fibre breaks the compliance of the material is altered there is no discernable effect on macroscopic strain as the breaks are isolated in the body of the composite. This means that although breaks can be detected by acoustic emission there is no measureable increase in macroscopic strain.

Figure 5: Comparison of simulated and experimental results of constant load tests, at $\mathbf{8 0 \%}$ failure stress, with unidirectional specimens loaded in the fibre direction.

\section{Application to the behaviour of a composite pressure vessel}

The above model can be applied to filament wound structures, such as tubes; however our immediate interest is in understanding the behaviour of composite pressure vessels. So as to extend the above approach to a pressure vessel, the structure has been modelled using finite elements, as shown in Figure 6. 
Figure 6: A finite element representation of a composite pressure vessel

Calculating the stress fields in the pressure vessel and applying the above multi-scale model allows damage accumulation to be calculated as a function of time and pressure.

Figure 7 shows a simulation of a monotonic burst test on a pressure vessel with a fibre volume fraction of $64 \%$. The dotted line shows the initial linear curve of pressure related to radial expansion of the vessel. The open circles represent points calculated by the model of pressure versus radial expansion showing the deviation from linearity corresponding to the beginning of mechanical instability. The filled circles represent the average number of fibre breaks in the RVEs, taken over the entire pressure vessel. Very few fibres are broken in plies which are not at $90^{\circ}$ with respect to the long axis of the vessel and even in the fretting layer, the low number emphasises that failure is due to localised coalescence of breaks.

Figure 7: Simulated burst tests a carbon fibre epoxy resin composite pressure vessel, with a fibre volume fraction of $\mathbf{6 4 \%}$. The open dotted line shows the initial linear curve of pressure related to radial expansion of the vessel. The open circles represent points calculated by the model of pressure versus radial expansion showing the deviation from linearity corresponding to the beginning of mechanical instability. The filled circles represent the average number of fibre breaks in the RVEs, taken over the entire pressure vessel. The low number emphasises that failure is due to localised coalescence of breaks.

The point at which the curve begins to deviate significantly from linearity is taken to show when fibre breaks begin to interact and coalesce, resulting in a physical instability of the pressure vessel, as has been shown experimentally by high resolution tomography [22].

Figure 8 shows a simulation of the accumulation of average number of fibre breaks in each RVE taken over the whole pressure vessel when it is held at $50 \mathrm{MPa}$, The pressure vessel is designed to have a burst pressure of approximately $80 \mathrm{MPa}$ The rate of damage can be seen to fall with time. Again, the average numbers are low as ultimate failure is due to clustering of fibre breaks rather than the overall failure rate reaching a critical level. The number of fibres in the fretting plies at $90^{\circ}$ to the major axis of the pressure vessel is approximately $100 \times 10^{6}$. Fibres in off-axis plies do not significantly contribute to numbers of fibre failures or ultimate burst strength. The result reflects the experimental curves obtained experimentally on pressure vessels and published elsewhere (16).

Figure 8 Simulated fibre failures in a pressure vessel, designed to fail at $80 \mathrm{MPa}$, pressurised to $50 \mathrm{MPa}$ and then maintained at that constant pressure showing the average number of fibre breaks in the RVEs as a function of time. The rate of damage can be seen to reduce with time. The number of fibres in the $90^{\circ}$ plies, which control the burst pressure, is approximately one hundred million. Off-axis plies do not contribute significantly to the number of fibre failures or burst pressure.

Figure 8 shows that the damage accumulated in the composite pressure vessel increases with time, however, this damage consists of regions of fibre breaks isolated by the effect of load
Formatted: Font: Not Bold

Formatted: Font: Not Bold Formatted: Font: Not Bold
Deleted: in a

Deleted: when it is held at 50MPa

Deleted: The crosses represent the calculated values and the continuous line the curve fitted to these points.

Formatted: Superscript
Deleted: Figure 8 Simulated fibre failures in a pressure vessel maintained at a constant pressure of 50MPa.II 
Figure 9: Fibre breaks occurring in a pressure vessel during pressurisation and then during the maintenance of the pressure constant. The curve for a pressure of $50 \mathrm{MPa}(500$ bars) shown in the previous figure is reduced because of the reduction in scale. The point of inflection seen on the curve for $70 \mathrm{MPa}(700$ bars) reveals the point at which regions of damage begin to interact and the pressure vessel is no longer reliable (21).

\section{Proof testing of composite pressure vessels}

The above analysis, together with the experimental results can be used to assess the state of damage of a pressure vessel even if it has not been previously monitored, providing that its loading history is known. Acoustic emission has been used by other authors to detect damage in carbon fibre composite pressure vessels (23) however usually to detect accidental damage rather than to predict future behaviour. Figures 8 and 9 reveal the state of damage as a function of time for a pressure vessel held at a steady load. If the loading pattern to which the

| pressure vessel has been subjected and over what period of time, is known, the results of this and previous studies have shown that the acoustic emission produced by the failure of the carbon fibres will follow a similar curve. The manner in which random fibre breaks have been induced in service is not of concern as it is the fibre break density which governs the acoustic activity when the pressure vessel is subjected to a constant pressure test. A master curve can therefore be drawn, using the above model, for an ideal pressure vessel which accumulates damage in this way and arrives at its inflexion point, or state of instability, exactly at the end of the period required; say twenty years. Any pressure vessel subjected to a similar load history will lie on such a curve and the question which needs to be answered is, will its curve reach the point of instability before or after that of the master curve. The proof test consists of subjecting the pressure vessel to a constant pressure equal to the maximum in-service pressure and monitoring the acoustic emission rate over a given period, of say twenty four hours. If the inflection point of the pressure vessel is greater than the required service life then the curve on which the pressure vessel lies will be under that of the master curve. A comparison of the numbers of emissions over the same period, of say twenty four hours will give a smaller number of emissions for the pressure vessel under test than that predicted by the master curve. In this case the vessel can remain in service. If not it should be removed. This comparison of curves is shown schematically in Figure 10 (3). The proposed test does not require knowledge of the above model, which however will allow the master curve to be determined for a given series of pressure vessels. The model can also be extended to predict the critical damage level corresponding to the point of inflexion. The test would involve simply monitoring damage by 
the acoustic emission technique for a predetermined time and a comparison with the results predicted for the master curve. This could be automated to make the test simpler.

Figure 10: The comparison of the acoustic activity, representing fibre failures, between a pressure vessel in service and the master curve of an ideal vessel which takes exactly the service life required to reach its point of instability.

\section{Conclusion}

The accumulation of fibre breaks in unidirectional composites has been accurately modelled. A simplified multiscale finite element approach has been employed in which the RVE takes into account the stochastic characteristics of fibre breaks, the variation of Weibull moduli due to finite numbers of fibres being considered, the load transfer through the matrix and its viscoelastic behaviour, as well as interfacial debonding. It has been shown that this model accurately describes the experimental results obtained in monotonic tensile tests and also in steady load tests. The model allows the scatter to be expected in experimental tests to be determined.

The use of parallel computation has allowed a multiscale model to be developed that allows the behaviour of filament wound structures and in the present study the whole of the pressure vessels to be described. The analogy between the behaviour of unidirectional composites and that of the reinforcements in the pressure vessels has been justified by a comparison between experimental and theoretical results. It has been shown that when the damage density is such that random fibre breaks are no longer isolated in the matrix but that an interaction begins to occur between damaged regions, the rate of damage begins to accelerate. This represents a point of physical instability which determines the limit of the safe use of a pressure vessel.

The proof testing technique which is proposed is based on an understanding of the real failure processes controlling the stability of the pressure vessel, rather than relying on intuition or models based on the behaviour of metals. The technique is simple to implement and therefore of low cost. It should allow this technology to progress at a faster rate than hitherto by optimising composite pressure design and allowing a reliable evaluation of residual lifetimes to be made.

\section{Acknowledgement}

The authors wish to thank Dr. D.H. Allen, Dean of Engineering at the University of Nebraska, Lincoln, USA for his support and valuable suggestions as well as Mr.S.Camara, of the same university, for help with the study and for the supply of some of the figures.

\section{References}

1. L.N. Phillips, 'Carbon fibre reinforced plastics. The first fifteen years' Plastics and Rubber International $3, \overline{6}(1978)$ pp. $239-243$.

2. A. Kelly 'Strong Solids' Clarendon Press, Oxford. 1963.

3. A.R.Bunsell 'Composite pressure vessels supply the answer to transport problems' Reinforced Plastics 50 (2006) pp.38-41.

Deleted: e

Deleted: L.N

Deleted: (1963)

Formatted: Font: Italic 
4. H.L. Cox 'The elasticity and strength of paper and other fibrous materials' British journal of applied physics, 12 (1951) pp.72-79.

5. B.W. Rosen 'Tensile failure of fibrous composites' AIAA Journal, 2 (1964) pp.1985-1991.

6. C. Zweben. 'Tensile failure of fiber composites' AIAA Journal, 6 (1968) pp.23252331 .

7. M.R. Nedele and M.R. Wisnom 'Three dimensional finite analysis of the stress concentration at a single fibre break' Composites science and technology, 41 (1991) pp.237-256.

8. C.M. Landis I.J. Beyerlein and R.M. McMeeking 'Micromechanical simulation of the failure of fiber reinforced composites' $\mathrm{J}$ mechanics and physics of solids, 48 (2000) pp.621-648.

9. J.M. Lifschitz and A. Rotem 'Time-dependent longitudinal strength of unidirectional fibrous composites' Fibre science and technology, 3 (1970) pp.1-20.

10. F. Feyel 'A multilevel finite element method $\left(\mathrm{FE}^{2}\right)$ to describe the response of highly non-linear structures using generalized continua' Computer methods in applied mechanics and engineering, 193 (2003) pp.3233-3244.

11. F.V. Souza, D.H. Allen and Y.R. Kim 'Multiscale model for predicting damage evolution in composites due to impact loading' Composites science and technology, 68 (2008) pp.2624-2634

12. A.S.Argon, Fracture: strength and toughness mechanisms. In: Comprehensive Composite Materials, vol. 1.24. Ed. A. Kelly, C. Zweben, .Pergamon, Oxford, pp. 763-802, 2000.

13. W.A.Curtin, Stochastic damage evolution and failure in fiber-reinforced composites. In: Advances in Applied Mechanics, vol. 36. Academic Press, London, pp.163-253, 1999.

14. S.L.Phoenix, I.J.Beyerlein, , 2000. Statistical strength theory for fibrous composite materials. In: Comprehensive composite materials, vol. 1.19. Pergamon, Oxford, pp. 559-639.

15. S. Blassiau, A. Thionnet and A.R. Bunsell 'Micromechanisms of load transfer in a unidirectional carbon-epoxy composite due to fibre failures, Part 1: Micromechanisms and 3D analysis of load transfer, the elastic case' Composite structures, 74 (2006) pp.303-318.

16. S. Blassiau, A. Thionnet and A.R. Bunsell 'Micromechanisms of load transfer in a unidirectional carbon-epoxy composite due to fibre failures, Part 2: Influence of viscoelastic and plastic matrices on the mechanism of load transfer' Composite structures, 74 (2006) pp.319-331.

17. S. Blassiau, A. Thionnet and A.R. Bunsell 'Micromechanisms of load transfer in a unidirectional carbon-epoxy composite due to fibre failures, Part 3: 'Multiscale reconstruction of composite behaviour' Composite structures, 83 (2008) 312-323

18. C. Baxevanakis 'Comportement statistique à rupture des composites stratifiés' Doctorate thesis Ecole des Mines de Paris (1994).

19. S. Blassiau, A.R. Bunsell, and A. Thionnet 'Damage accumulation processes and life prediction in unidirectional composites' Proc.R.Soc.A463 (2007) 1135-1152.

20. M.-H. Berger and D. Jeulin, 'Statistical analysis of the failure stresses of ceramic fibres: Dependence of the Weibull parameters on the gauge length, diameter variation and fluctuation of defect density' J Mat Sci 38 (2003) pp.2913 - 2923.

21. S. Camara, 'A 3D Multiscale Finite Element Analysis of Damage Accumulation in Carbon Fiber Unidirectional Composites,: Applied to Pressure Vessels': MS thesis Nebraska University Lincoln, 2009.

Formatted: Font: Italic

Deleted: W.A.
Deleted: 1999.
Formatted: Font: Italic
Deleted: S.L.,
Deleted: I.J.
Formatted: Font: Italic

Formatted: Font: Italic

Formatted: Bullets and Numbering

Formatted: English (U.K.)

Formatted: English (U.K.) 
22. A.E.Scott, M.Clinch, W.Hepples, N.Kalantzis, I.Sinclair, S.M.Spearing 'Advanced micromechanical analysis of highly loaded hybrid composite structures' Proceedings of ICCM-17, Edinburgh, 29-31 July 2009.

23. K.S.Downs and M.A.Hamstad, 'Acoustic emission from depressurization to detect/evaluate significance of impact damage to graphite/epoxy pressure vessels' J Comp.Mat. 32, 3 (1998) pp.258-307.

Deleted: $\Phi$ 
2

3

4

5

6

7

8

9

10

11

12

13

14

15

16

17

18

19

20

21

22

23

24

25

26

27

28

29

30

31

32

33

34

35

36

37

38

39

40

41

42

43

44

45

46

47

48

49

50

51

52

53

54

55

56

57

58

59

60

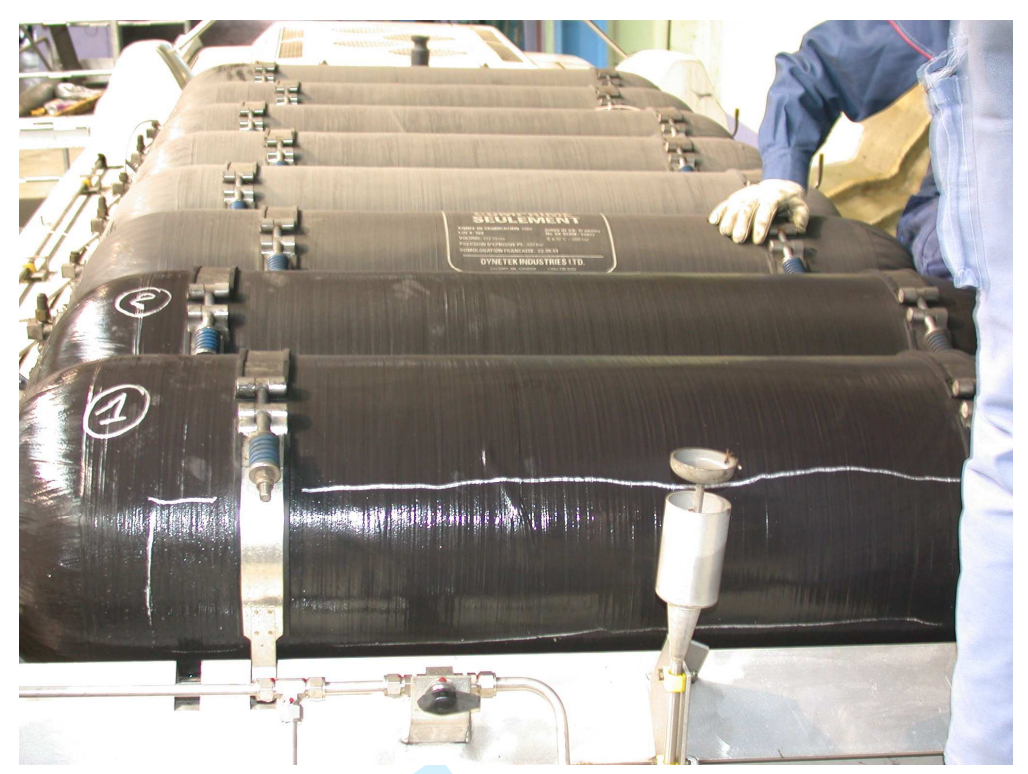

Figure 1: Natural gas cylinders made from carbon fibre reinforced epoxy resin on the roof of a bus.

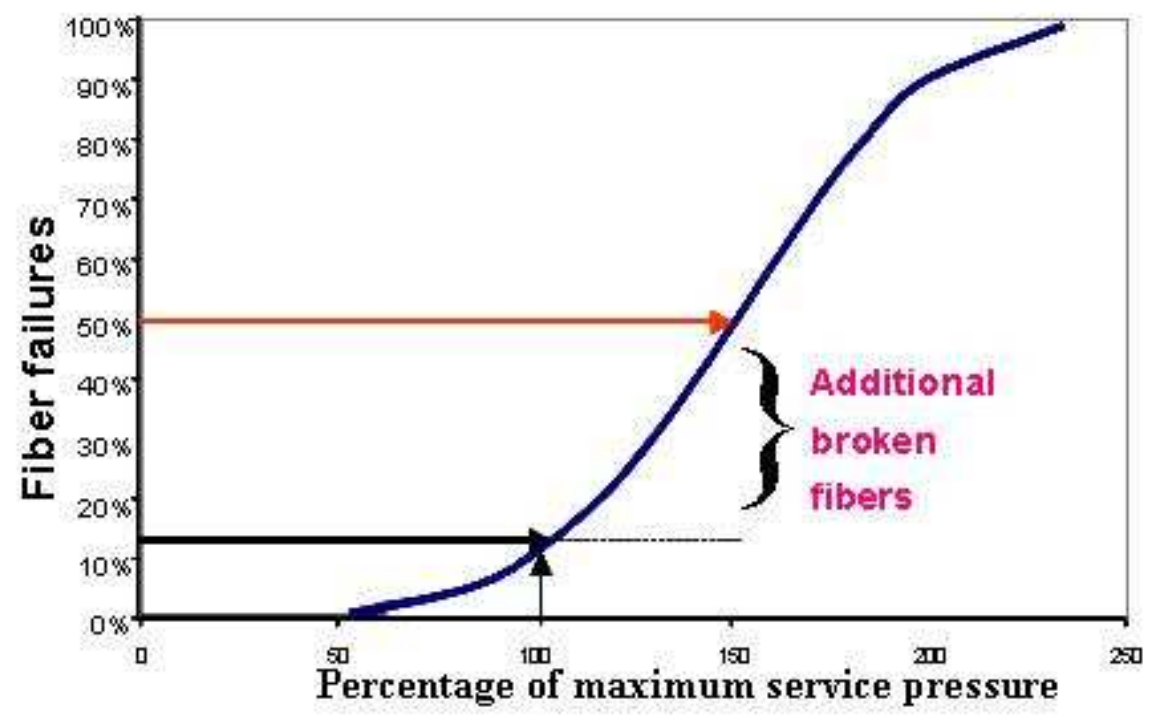

Figure 2: A hydraulic test would greatly increase the number of broken fibres in the pressure vessel. 


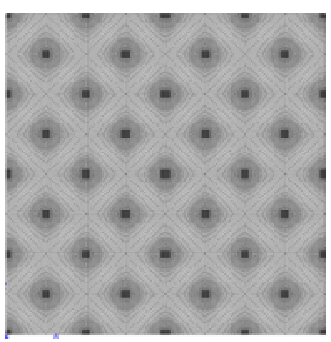

Cell CS32

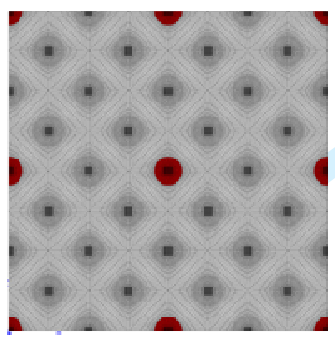

Cell C8

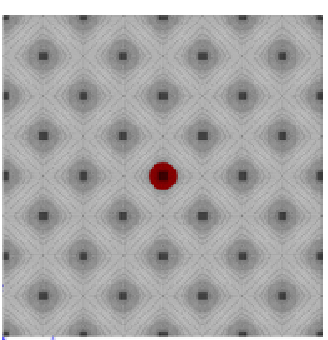

Cell C32

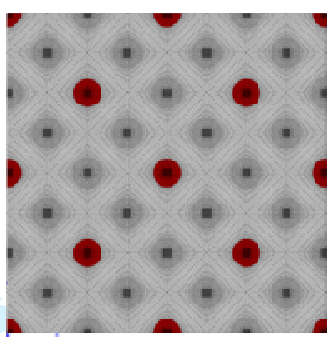

Cell C4

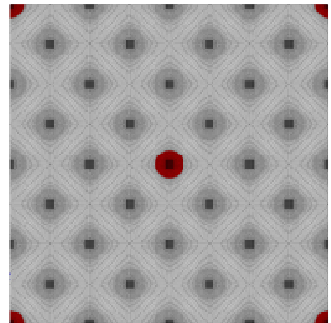

Cell C16

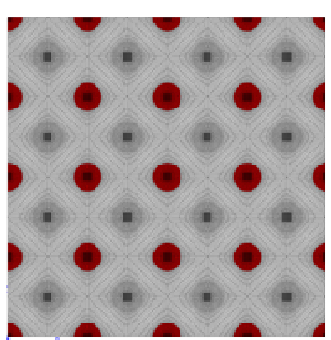

Cell C2

Figure 3: Model of the failure process on the scale of the carbon fibres.

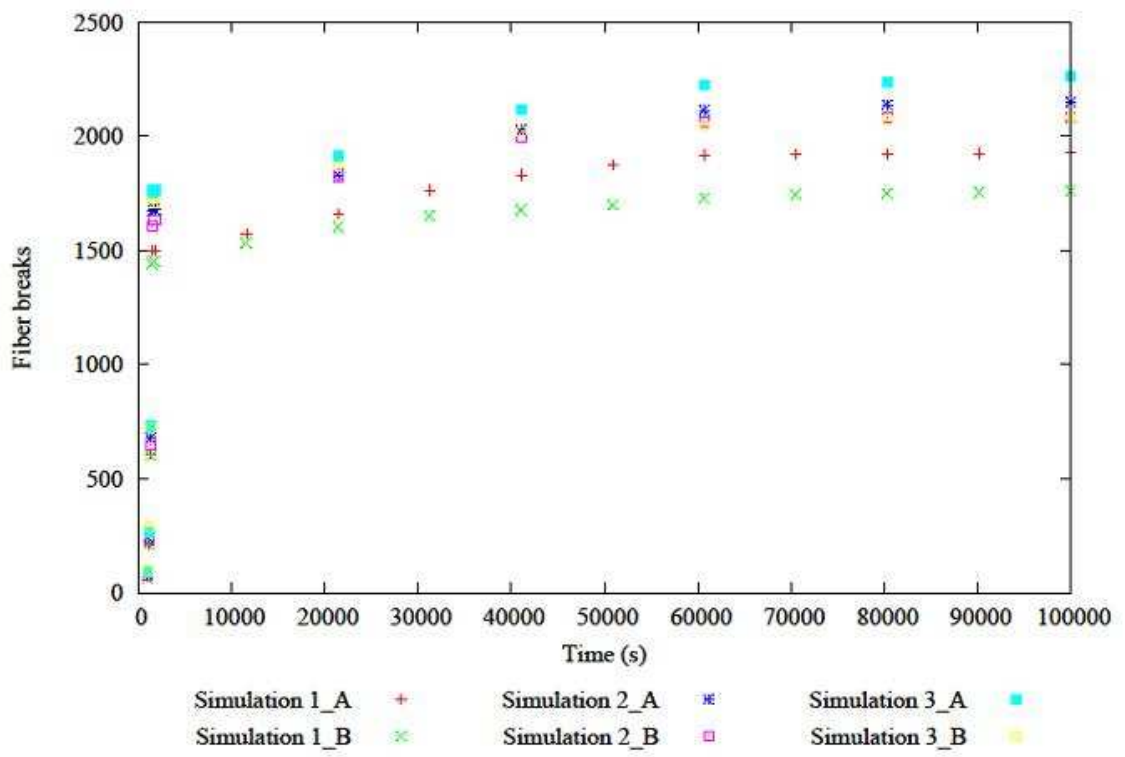

Figure 4: Simulated fibre failures in unidirectional composites loaded to $80 \%$ of failure load showing scatter due to the stochastic nature of fibre strengths and also variations in Weibull moduli between the $A$ and $B$ curves. 
1

2

3

4

5

6

7

8

9

10

11

12

13

14

15

16

17

18

19

20

21

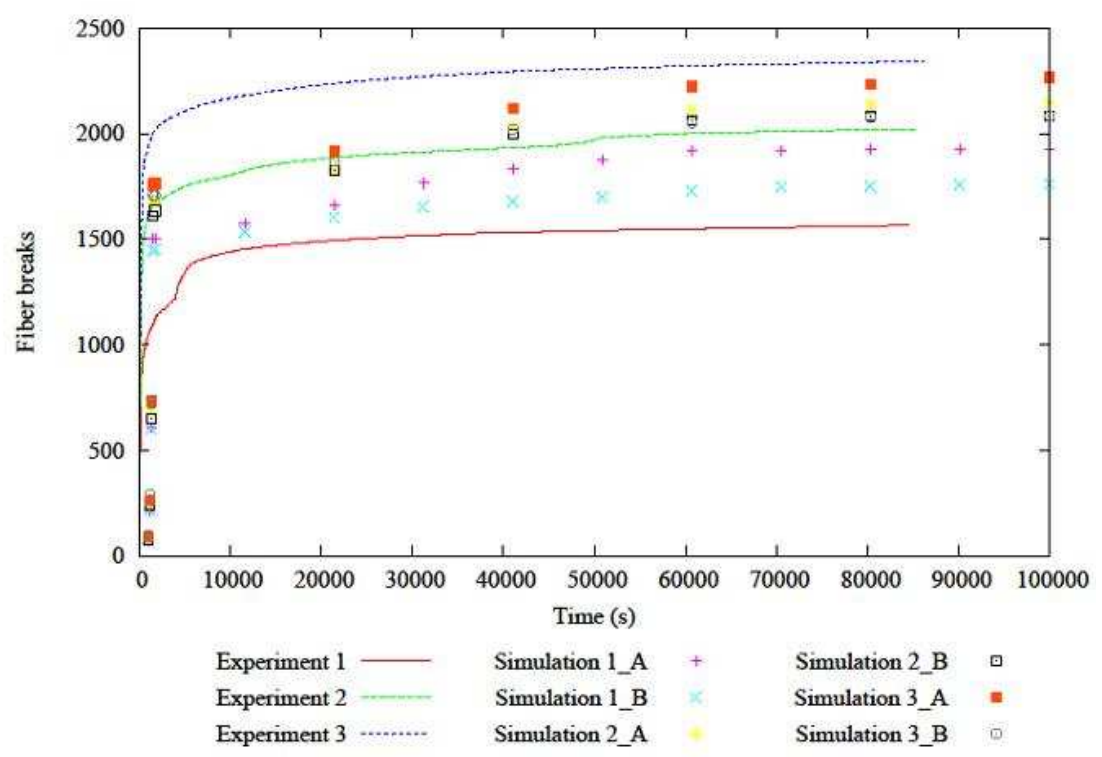

Figure 5: Comparison of simulated and experimental results of constant load tests, at $80 \%$ failure stress, with unidirectional specimens loaded in the fibre direction.

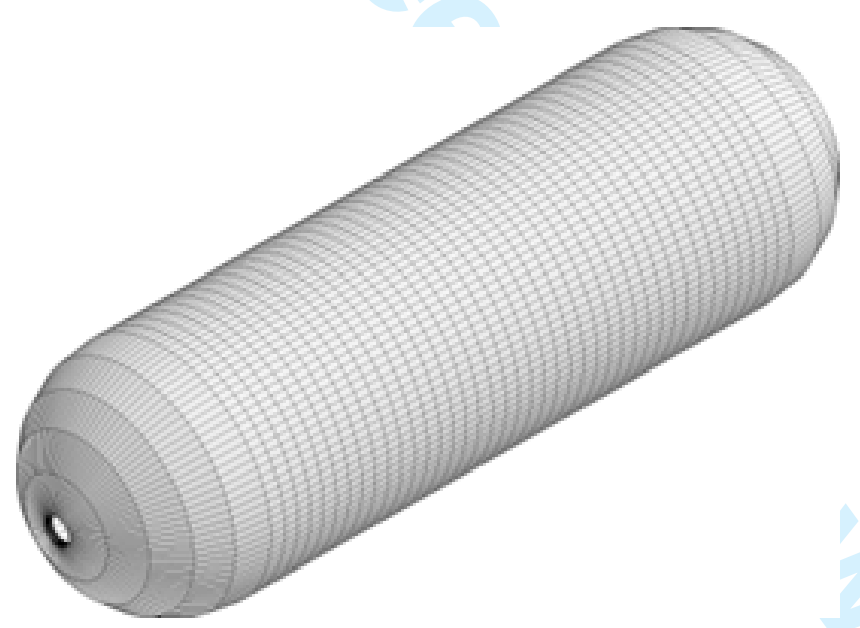

Figure 6: A finite element representation of a composite pressure vessel 


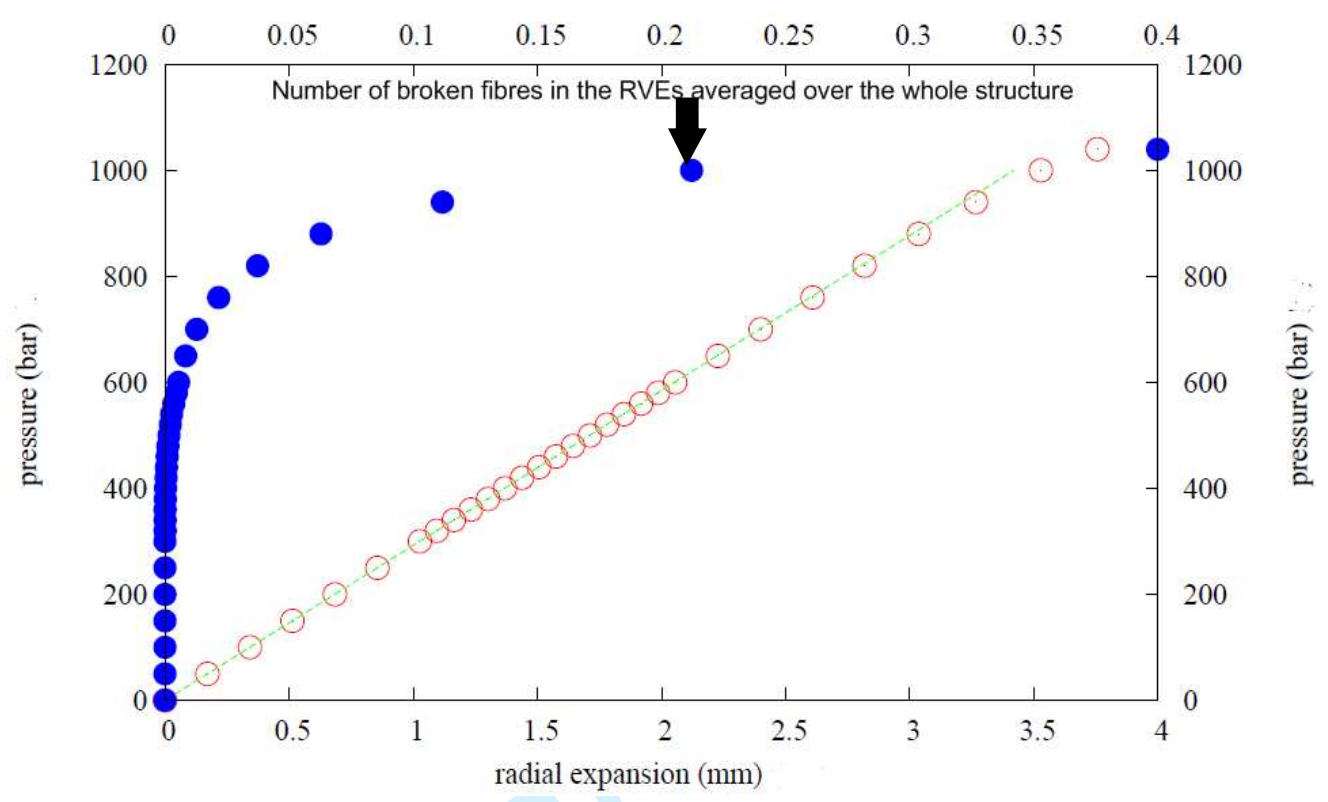

Figure 7: Simulated burst tests a carbon fibre epoxy resin composite pressure vessel, with a fibre volume fraction of $64 \%$. The open dotted line shows the initial linear curve of pressure related to radial expansion of the vessel. The open circles represent points calculated by the model of pressure versus radial expansion showing the deviation from linearity corresponding to the beginning of mechanical instability. The filled circles represent the average number of fibre breaks in the RVEs, taken over the entire pressure vessel. The low number emphasises that failure is due to localised coalescence of breaks. 


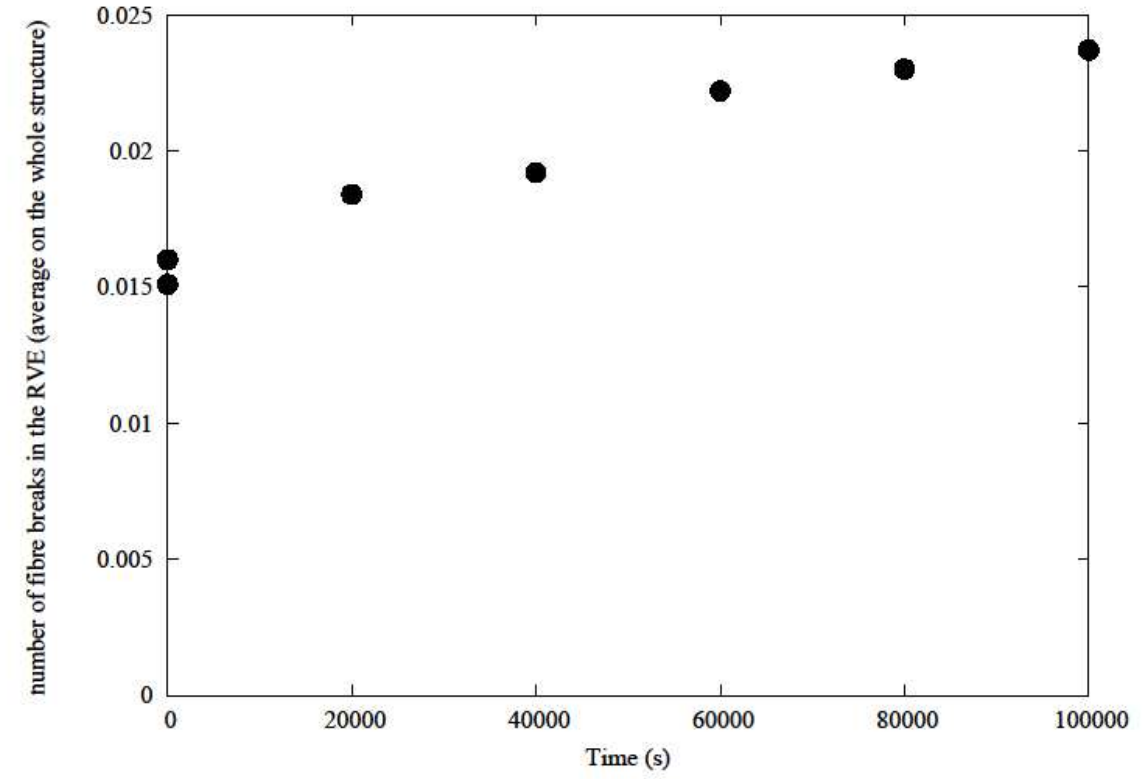

Figure 8 Simulated fibre failures in a pressure vessel, designed to fail at $80 \mathrm{MPa}$, pressurised to $50 \mathrm{MPa}$ and then maintained at that constant pressure showing the average number of fibre breaks in the RVEs as a function of time. The rate of damage can be seen to reduce with time. The number of fibres in the $90^{\circ}$ plies, which control the burst pressure, is approximately one hundred million. Off-axis plies do not contribute significantly to the number of fibre failures or burst pressure.
Deleted: <sp >

Deleted: Figure 8 Simulated fibre failures in a pressure vessel maintained at a constant pressure of 50MPa. II 
Figure 9: Fibre breaks occurring in a pressure vessel during pressurisation and then during the maintenance of the pressure constant. The curve for a pressure of $50 \mathrm{MPa}(500$ bars) shown in the previous figure is reduced because of the reduction in scale. The point of inflection seen on the curve for $70 \mathrm{MPa}(700$ bars) reveals the point at which regions of damage begin to interact and the pressure vessel is no longer reliable (21). 


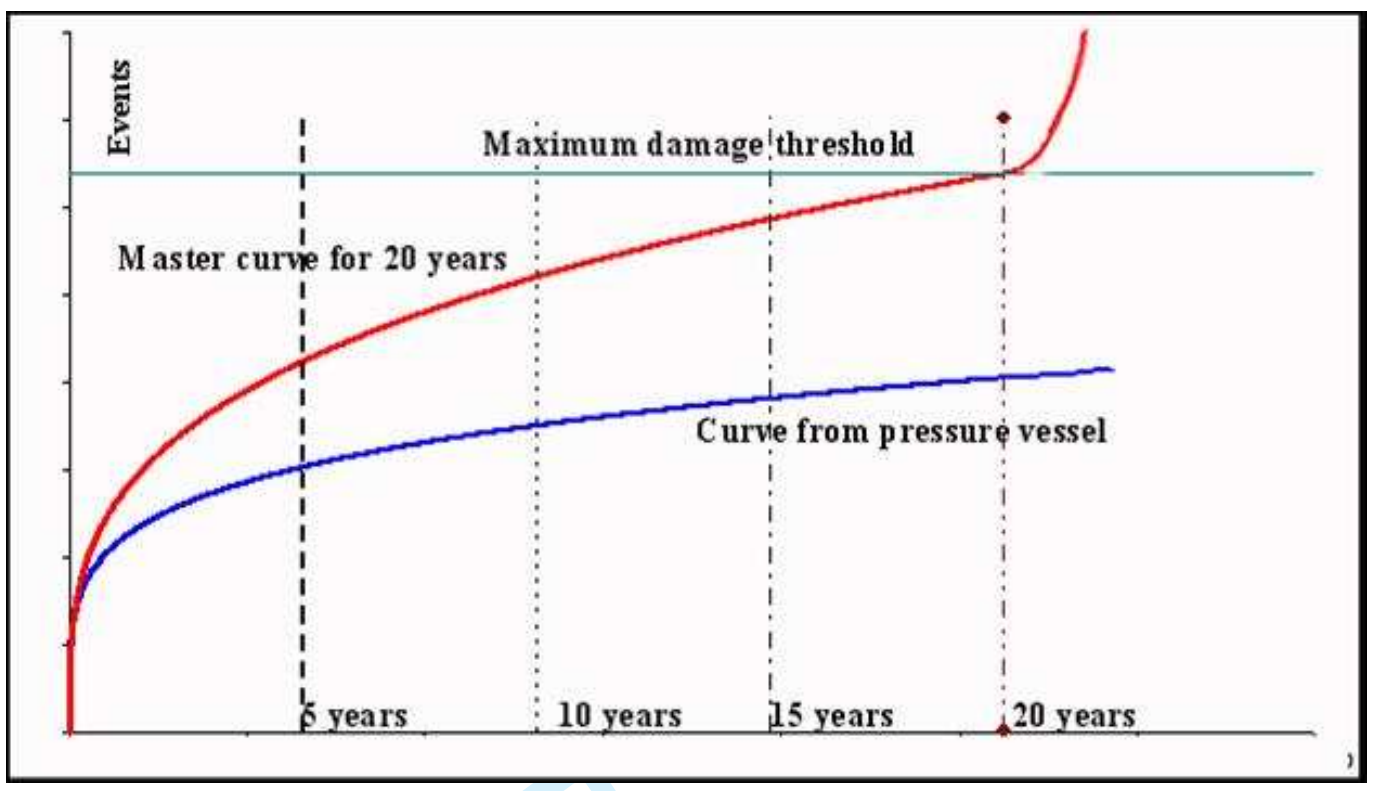

Figure 10: The comparison of the acoustic activity, representing fibre failures, between a pressure vessel in service and the master curve of an ideal vessel which takes exactly the service life required to reach its point of instability. 